\title{
Loncastuximab Tesirine
}

National Cancer Institute

\section{Source}

National Cancer Institute. Loncastuximab Tesirine. NCI Thesaurus. Code C125549.

An antibody-drug conjug ate (ADC) consisting of an anti-CD19 humanized monoclonal antibody conjug ated, via a cleavable linker comprised of valine-alanine and maleimide, to a cytotoxic, cross-linking agent pyrrolobenzodiazepine (PBD) dimer, which targets DNA minor grooves, with potential antineoplastic activity. Upon administration, the monoclonal antibody portion of loncastuximab tesirine targ ets the cell surface antigen CD19 on various cancer cells. Upon antibody/antigen binding and internalization, the cytotoxic PBD moiety is released. The imine groups of the PBD moiety bind to the N2 positions of guanines on opposite strands of DNA. This induces interstrand cross-links in the minor groove of DNA and inhibits DNA replication, which inhibits the proliferation of CD19-overexpressing tumor cells. CD19, a transmembrane receptor and tumorassociated antigen (TAA), is expressed on a number of B-cell-derived cancers. 